\title{
P02-3-41 Poster session
}

\section{Genetic deletion of the Kvbeta subunit leads to altered cardiac levels of SLC41 a sodium-magnesium transporter}

\author{
Jared Tur, Kalyan C Chapalamadugu, Srinivas M Tipparaju \\ College of Pharmacy, University of South Florida, USA
}

Magnesium is the second most prominent intracellular cation and the fourth most abundant within the human body clinical research has demonstrated a striking inverse relation to circulatory levels of magnesium and risk for cardiac injury. While clinical evidence has highlighted the cardiac benefits of magnesium both identification and regulation of these specific magnesium channels remain largely unknown. Isolated cardiomyocytes demonstrate profound magnesium influx/efflux behaviors addition of magnesium during and prior to cardiac injury such as ischemia reperfusion was demonstrated to reduce ischemic injury. While evident magnesium is entering the cardiomyocyte the identification of the specific channels responsible remains largely unknown. Recently published SLC41a3 and its subunits a1 and a2 were found to code a sodium magnesium transporter. Further research demonstrated significant mRNA levels of SLC41a3 within murine hearts along with expression levels of a1 and a2 as well. A recent microarray study performed in Kvbeta2 KO hearts demonstrated a significant decrease in SLC41a3 mRNA expression compared with wildtype control. Further protein expression validated a significantly decrease in expression level of SLC41a3 in KO hearts. This work may indicate that the Kvbeta2 subunit acts as a regulator to SLC41a3 previous literature has identified Kvbeta2 as a novel protein interactor with SLC39a1 a zinc transporter and family domain to SLC41a. Monophasic action potential recordings have demonstrated a significant reduction in AP duration values in wildtype hearts perfused with magnesium $\mathrm{MgSO} 4$ while $\mathrm{KO}$ demonstrate little to no alterations in AP duration post magnesium exposure. Based on our preliminary data we hypothesis that Kvbeta2 plays significant roles in SLC41a3 expression as well as function within the heart. Further functionality will be tested in isolated cardiomyocytes from wildtype and $\mathrm{KO}$ hearts to test the influx and efflux of magnesium within these isolated cells to understand how Kvbeta 2 absence alters not only expression levels of but channel activity as well. 